\title{
A enfermagem no manejo da dor em unidades de atendimento de urgência e emergência
}

Paulo Eduardo Pires de Oliveira ${ }^{1}$, Lilian Varanda Pereira ${ }^{2}$, Nilde Resplandes Santos ${ }^{3}$, Layz Alves Ferreira Souza ${ }^{4}$

\footnotetext{
${ }^{1}$ Enfermeiro. Enfermeiro da Unidade de Terapia Intensiva do Hospital Anis Rassi. Goiânia, GO, Brasil. E-mail: eduardopepo@hotmail.com.

${ }^{2}$ Enfermeira, Doutora em Enfermagem Fundamental. Professora Adjunto da Faculdade de Enfermagem da Universidade Federal de Goiás. Goiânia, GO, Brasil. E-mail: lilianvaranda7@gmail.com.

${ }^{3}$ Enfermeira, Mestre em Enfermagem. Enfermeira do Hospital de Clínicas da Universidade Federal de Goiás. Goiânia, GO, Brasil. E-mail: nildes_resplandes@hotmail.com.

${ }^{4}$ Enfermeira, Mestre em Enfermagem. Discente do Programa de Pós-Graduação em Enfermagem, nível Doutorado, da UFG. Enfermeira da Clínica Cirúrgica do Hospital de Clínicas da Universidade Federal de Goiás. Goiânia, GO, Brasil. E-mail: layzenf@gmail.com.
}

Recebido: 21/08/2015.

Aceito: 04/12/2015.

Publicado: 30/06/2016.

Como citar esse artigo:

Oliveira PEP, Pereira LV, Santos NR, Souza LAF. A enfermagem no manejo da dor em unidades de atendimento de urgência e emergência. Rev. Eletr. Enf. [Internet]. 2016 [acesso em: / ];18:e1171. Disponível em: http://dx.doi.org/10.5216/ree.v18.37309.

\section{RESUMO}

A enfermagem deve manejar a dor no atendimento em urgência e emergência, baseando-se em evidências científicas, mas isso parece apresentar obstáculos. Objetivou-se identificar e analisar as estratégias de manejo da dor e as barreiras para sua utilização entre as categorias profissionais da enfermagem nos serviços de urgência e emergência. Revisão integrativa, com buscas no LILACS, SciVerse Scopus, PUBMED e na literatura cinzenta do Google acadêmico. Foram selecionados 15 artigos publicados entre 2012 e 2015 . A enfermagem avaliou as características da dor por meio de anamnese e uso de instrumentos. As intervenções restringiram-se prioritariamente às farmacológicas, apontando a necessidade de associá-las às não farmacológicas. A monitorização da dor ainda é incipiente. Os profissionais necessitam avançar no uso de instrumentos válidos para avaliação, intervenção e monitoramento da dor, com o propósito de vencer as barreiras que profissionais, clientes e gerentes das unidades de urgência e emergência encontram para sistematizar o seu manejo. Descritores: Enfermagem em Emergência; Dor; Manejo da Dor.

\section{INTRODUÇÃO}

A dor é uma das principais queixas de usuários atendidos em unidades de urgência e emergência $(\text { UUEs) })^{(1)}$. Embora o adequado alívio dessa experiência seja um direito humano ${ }^{(2-3)}$, a produção de conhecimento sobre a atuação do enfermeiro na avaliação, alívio e monitoramento da dor nessas unidades ainda é escasso ${ }^{(4-7)}$. Uma busca, nas bibliotecas, de revisões sistemáticas do Cochrane e Joanna Briggs 
Institute encontrou apenas uma revisão (sem metanálise) a respeito das intervenções de enfermagem em pacientes adultos com dor crônica ${ }^{(8)}$. No entanto, tal trabalho não abordou questões específicas do manejo da dor no contexto de urgência e emergência, que se caracteriza prioritariamente por episódios de dor aguda. Também não foram abordadas as barreiras para a execução desse manejo.

Essa escassez de estudos dificulta a disseminação de conhecimento sobre a importância da avaliação da dor, das estratégias disponíveis para o seu controle e da transposição de barreiras para a prática de enfermagem baseada em evidências. Ademais, o alívio inadequado da dor aguda pode aumentar as catecolaminas, que geram taquicardia, e também elevar a pressão arterial e o consumo de oxigênio pelo miocárdio. Consequentemente, podem ocorrer disfunções ventriculares e isquemia do miocárdio. A dor aguda também induz a produção de hormônios, como cortisol e glucagon, que aumentam os riscos de as pessoas desenvolverem resistência à insulina, hiperglicemia, hipercoagulabilidade e disfunções imunológicas ${ }^{(7,9)}$. Tais alterações podem agravar o quadro clínico do indivíduo em situação de urgência ou emergência.

Durante o atendimento em uma UUE, devem ser avaliadas as queixas e os fatores agravantes\atenuantes e concomitantes da dor; os antecedentes pessoais e familiares e os indicadores de desconforto advindos dessa experiência, que devem ser mensurados por meio de instrumentos pelos quais o paciente possa expressar-se e que subsidiem a escolha da melhor terapêutica analgésica ${ }^{(9-12)}$.

Após a avaliação da experiência dolorosa, o enfermeiro deve ser capaz de executar e prescrever intervenções de enfermagem que contribuam para o alívio da dor, além de administrar os analgésicos prescritos e monitorar o alívio e as complicações advindas da analgesia ${ }^{(9)}$. No entanto, estudos ${ }^{(4-6,10,13-15)}$ mostram haver desconhecimento e barreiras quanto à atuação da enfermagem no adequado manejo da dor em UUEs.

Portanto, considerando que a maior parte das situações clínicas de atendimento em UUEs envolve a presença de dor, que essa experiência não pode ser negligenciada no processo de avaliação e intervenção e que investigações sobre o manejo da dor realizado pela enfermagem nessas unidades são escassas, esse estudo foi realizado com o propósito de contribuir para a aquisição de conhecimentos sobre a atuação da enfermagem no manejo da dor em UUEs. Para tal, estabeleceu-se como objetivo identificar e analisar as estratégias para o manejo da dor, bem como as barreiras para sua utilização entre as categorias profissionais da enfermagem, nos serviços de urgência e emergência.

\section{MÉTODO}

Revisão integrativa da literatura, realizada entre janeiro e dezembro de 2015. O percurso metodológico, elaborado com base em propostas de outros autores ${ }^{(16-17)}$, pode ser observado na Figura 1. 
1. Definicão da pergunta de pesquisa: “Quais estratégias os profissionais de enfermagem utilizam para o manejo da dor em situações de urgência e emergência e quais as barreiras que inviabilizam o uso dessas estratégias?"

2. Seleção dos Descritores Controlados em Saúde (DeCS/MESH) e bibliotecas/bases/fontes de dados para a busca na literatura (LILACS, PUBMED, SCOPUS, Google Acadêmico)

3. Estabelecimento dos critérios de inclusão (período de publicação entre janeiro de 2002 e dezembro de 2015, publicados em português, inglês ou espanhol, que abordassem a atuação da equipe de enfermagem no manejo da dor em UUEs) e exclusão (trabalhos sem os resumos disponíveis, os relatos de experiência, artigos de reflexão e teses ou dissertações)

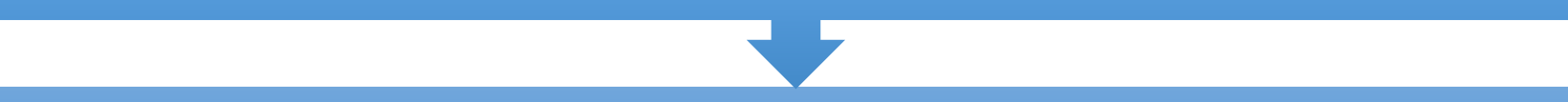

4. Busca nas fontes de dados ( $n=50.366)$ e seleção de resumos $(n=53)$

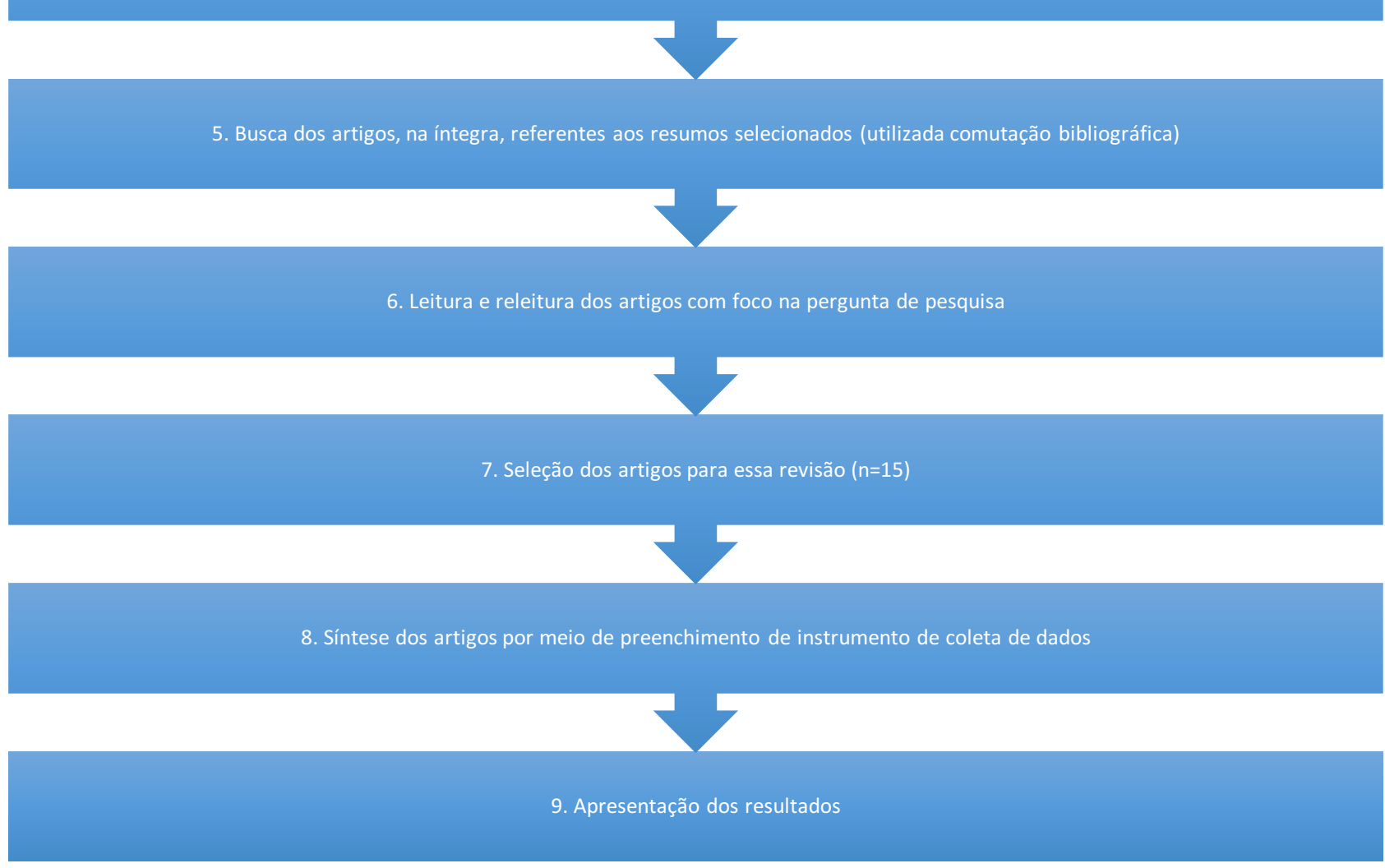

Figura 1: Percurso metodológico da revisão integrativa realizada. Goiânia, GO, Brasil, 2015.

Para a elaboração da pergunta de pesquisa, identificou-se a população alvo, as variáveis de interesse e os resultados esperados (Estratégia PVO; adaptação da estratégia PICO, recomendada pela Bireme para situações em que não se pesquisa uma intervenção específica) ${ }^{(18)}$. A população de interesse constituiu-se em enfermeiros, técnicos e auxiliares de enfermagem de serviços de urgência e emergência. A variável de interesse foi o manejo da dor (avaliação, intervenção e/ou monitorização da dor) e as barreiras vivenciadas pelos profissionais de enfermagem durante esse manejo. Os resultados esperados foram as estratégias utilizadas pela equipe de enfermagem para avaliação da dor (uso de instrumentos, anamnese ou exame físico), tipos de intervenções utilizadas (farmacológicas e/ou não farmacológicas), acompanhamento da evolução clínica da dor (monitoramento da dor), relatos ou apontamentos sobre dificuldades encontradas 
pelos profissionais para a realização do adequado manejo da dor (barreiras para o manejo da dor). Assim, a pergunta de pesquisa foi: "Quais estratégias os profissionais de enfermagem utilizam para o manejo da dor em situações de urgência e emergência e quais as barreiras que inviabilizam o uso dessas estratégias?"

A busca foi realizada na base de dados da Literatura Latino-Americana e do Caribe em Ciências da Saúde (LILACS), SciVerse Scopus e no portal US National Library of Medicine National Institutes of HealthPubmed. Também foi utilizada a literatura cinzenta no Google Acadêmico, por permitir ampliar a busca ${ }^{(17)}$. Para busca no PubMed e SciVerse Scopus utilizou-se a busca controlada, por meio dos cruzamentos com o operador boleano and, os MESH utilizados foram Emergency Nurse, Pain, Measurement Pain, Management Pain, Emergency Treatament, Emergency Medical Service, Emergency Hospital Service, Based Evidence, Ambulances, Air Ambulance e Emergencies.

Nas demais bases de dados e no Google acadêmico, foram selecionados descritores controlados conforme os Descritores em Ciências da Saúde (DeCS - edição 2014) e realizados os seguintes cruzamentos entre Enfermagem em Emergência, Dor, Avaliação de Dor, Manejo de Dor, Medição de Dor, Tratamento de Emergência, Medicina de Emergência, Medicina de Emergência Baseada em Evidências, Serviços Médicos de Emergência, Serviços Hospitalares de Emergência, Resgate Aéreo, Ambulâncias, Socorristas.

Foram incluídos os artigos publicados entre janeiro de 2002 e 20 de dezembro de 2015, período posterior à Portaria GM/MS no 2048/2002, que regulamentou os sistemas estaduais de urgência e emergência no Brasil. Demais critérios de inclusão e exclusão podem ser observados na Figura 1.

O cruzamento dos descritores resultou em 34 trabalhos na LILACS, 35.733 no Google Acadêmico, 11.377 no PubMed e 3.170 no SciVerse Scopus. Os resumos foram avaliados pela aplicação do Teste de relevância I ${ }^{(16)}$. Quando selecionados, passaram por nova avaliação (Teste de Relevância II), pelos seguintes questionamentos: O estudo está voltado para a solução do problema específico que está sendo investigado? Ou seja, o estudo avalia de alguma forma como os profissionais de enfermagem realizam o manejo da dor em situação de urgência ou emergência e as barreiras vivenciadas nesse contexto?

Após esta etapa, foram selecionados os resumos e esses tiveram seus textos obtidos na íntegra, com auxílio do setor de comutação bibliográfica do Ministério da Saúde, sendo analisados por meio do Teste de Relevância Final ${ }^{(16)}$. Para cada artigo foi preenchido um formulário com respostas às seguintes indagações: 0 problema da pesquisa tem relação com o manejo da dor por profissionais de enfermagem em situações de urgência e emergência? O objetivo do estudo tem relação com a questão estudada pela revisão? A metodologia está bem descrita, de forma que possa ser reproduzida? A metodologia está adequada para o alcance dos objetivos? Os resultados são compatíveis com a metodologia utilizada no estudo? A aplicação dos resultados é possível na prática, sendo os benefícios superiores aos riscos potenciais e justificam os custos? Os artigos que atenderam às perguntas foram incluídos na revisão.

Foram selecionados para esta revisão oito artigos do PubMed, três do SciVerse Scopus, três do Google Acadêmico e um trabalho da LILACS, totalizando 15 estudos. Utilizou-se um instrumento elaborado pelos próprios autores que contemplou itens sobre a identificação do artigo, instituição sede do estudo, o tipo de 
publicação (área da saúde a qual pertence a publicação), características metodológicas (tipo de estudo, características da amostra estudada, características da dor avaliada, instrumentos de avaliação de dor estudados, questão da pesquisa), resultados obtidos no estudo, conclusões e a avaliação do rigor metodológico. As informações foram extraídas mediante exaustiva leitura.

Posteriormente, foram analisadas as características metodológicas dos estudos, classificando-os em seis níveis de evidências ${ }^{(19)}$ : Nível I- metanálise de múltiplos estudos controlados; Nível II- estudos individuais com delineamento experimental; Nível III- estudos com delineamento quase experimental, coorte ou casocontrole; Nível IV- estudo com delineamento não experimental, inclusive pesquisa qualitativa e estudos de caso; Nível V-relatório de casos ou dado obtido de forma sistemática, de qualidade verificável, ou dados de avaliação de programas; Nível VI- opinião de autoridades respeitáveis baseada na competência clínica ou opinião de comitês de especialistas.

\section{RESULTADOS}

Os achados evidenciaram que a maior parte dos estudos sobre a temática ainda se concentra na avaliação da dor do paciente; quando abordam intervenções, essas restringem-se prioritariamente à administração de medicamentos conforme prescrição médica e a monitorização desse processo ainda é incipiente, apontando fragilidades no manejo da dor em UUEs ${ }^{(4-6,10,13-15,20-25)}$.

As barreiras apontadas para a efetivação do manejo da dor relacionam-se ao próprio profissional (medo, insegurança, frustração, falta de empatia com cliente, entre outros), ao cliente (dificuldade em entender as escalas utilizadas, instabilidade clínica, relutância em aceitar opioide) ou às questões organizacionais da instituição, como deficiência nos processos de educação continuada sobre o tema, conflitos entre profissionais e gestores, alta demanda de pacientes, infraestrutura e organização do serviço ${ }^{(4-}$ $6,10,13-15,22,24,26-27)$

A síntese dessa revisão pode ser observada no Quadro 1. 
Quadro 1: Distribuição dos artigos selecionados $(n=15)$ segundo autores, ano e local de publicação, nível de evidência e síntese quanto aos resultados encontrados em relação ao manejo e barreiras para alívio da dor em UUEs. Goiânia, GO, Brasil, 2015.

\begin{tabular}{|c|c|c|c|c|}
\hline \multirow{2}{*}{$\begin{array}{c}\text { Autores/País/Ano/nível } \\
\text { de evidência }\end{array}$} & \multicolumn{3}{|c|}{ Manejo da dor } & \multirow{2}{*}{ Barreiras no manejo da dor } \\
\hline & Avaliação & Intervenção & Monitorização & \\
\hline $\begin{array}{l}\text { Callil e Pimenta }^{(7)} \\
\text { Brasil } \\
2005 \\
\text { IV }\end{array}$ & $\begin{array}{l}\text { Enfermeiros relataram avaliar a dor } \\
\text { por meio do relato do paciente } \\
\text { (81\%), agitação, fácies de dor e/ou } \\
\text { de conforto }(18,2 \%) \text {. }\end{array}$ & $\begin{array}{l}\text { Administrar medicamentos analgésicos } \\
\text { conforme prescrição médica. }\end{array}$ & $\begin{array}{l}\text { Avaliação da analgesia } \\
\text { por meio do relato da } \\
\text { presença ou ausência de } \\
\text { dor, após administração } \\
\text { do medicamento. }\end{array}$ & $\begin{array}{l}\text { Insegurança ou subadministração de analgésicos } \\
\text { opioides quando o cliente apresentava escore da } \\
\text { escala de Glasgow }<9 \text { e/ou presença de traumatismo } \\
\text { crânio-encefálico. }\end{array}$ \\
\hline $\begin{array}{l}\text { Stalnikowicz et al. } \\
\text { Israel } \\
2005 \\
\text { II }\end{array}$ & $\begin{array}{l}\text { Os enfermeiros utilizaram a Escala } \\
\text { Visual Analógica (EVA) no serviço de } \\
\text { triagem da UUE. }\end{array}$ & $\begin{array}{l}\text { Encaminhar o cliente para o atendimento } \\
\text { médico e administrar medicamentos } \\
\text { analgésicos prescritos. Casos prioritários } \\
\text { para o atendimento foram selecionados } \\
\text { com base no quadro clínico e na maior } \\
\text { intensidade de dor (EVA>7). }\end{array}$ & $\begin{array}{l}\text { A dor foi reavaliada 30- } \\
60 \text { minutos após a } \\
\text { medicação analgésica e } \\
\text { no momento da alta. } \\
\text { Verificou-se redução na } \\
\text { média dos escores de } \\
\text { dor (EVA 5-7). }\end{array}$ & Não apresentadas \\
\hline $\begin{array}{l}\text { Rampanjato et al. } \\
\text { África Central } \\
2007 \\
\text { IV }\end{array}$ & $\begin{array}{l}\text { Os enfermeiros utilizaram a Escala } \\
\text { Numérica de Dor (EN) no serviço de } \\
\text { triagem da UUE. }\end{array}$ & $\begin{array}{l}\text { Administrar medicamentos analgésicos } \\
\text { segundo protocolo da instituição, ainda na } \\
\text { triagem. Clientes com escores }>7 \text { na EN } \\
\text { receberam analgesia prioritariamente } \\
\text { quando comparados com clientes que } \\
\text { avaliaram sua dor como de menor } \\
\text { intensidade }(p<0,05) \text {. }\end{array}$ & Não apresentada & $\begin{array}{l}\text { Enfermeiros relataram sentir-se incapazes de avaliar } \\
\text { a dor adequadamente (80\%), e } 67 \% \text { admitiram } \\
\text { temor em administrar morfina. }\end{array}$ \\
\hline $\begin{array}{l}\text { Le May et al. } \\
\text { Canadá } \\
2009 \\
\text { II }\end{array}$ & Não apresentada & Não apresentada & Não apresentada & $\begin{array}{l}\text { Ausência ou deficiência de intervenções } \\
\text { educacionais para o manejo da dor podem } \\
\text { prejudicar a assistência de enfermagem em UUE. } \\
\text { Enfermeiros que receberam intervenções } \\
\text { educacionais sobre o tema melhoraram a } \\
\text { documentação da experiência dolorosa e o uso de } \\
\text { terapêutica analgésica não farmacológica. }\end{array}$ \\
\hline
\end{tabular}




\begin{tabular}{|c|c|c|c|c|}
\hline \multirow{2}{*}{$\begin{array}{c}\text { Autores/País/Ano/nível } \\
\text { de evidência }\end{array}$} & \multicolumn{3}{|c|}{ Manejo da dor } & \multirow{2}{*}{ Barreiras no manejo da dor } \\
\hline & Avaliação & Intervenção & Monitorização & \\
\hline $\begin{array}{l}\text { Van der Wulp et al. } \\
\text { Holanda } \\
2011 \\
\text { IV }\end{array}$ & $\begin{array}{l}\text { Os enfermeiros do serviço de } \\
\text { triagem de UUE não avaliavam } \\
\text { comportamentos de dor e limitavam- } \\
\text { se aos questionamentos } \\
\text { preconizados no protocolo de } \\
\text { Manchester e à aferição dos Sinais } \\
\text { Vitais para inferências sobre a } \\
\text { experiência dolorosa. }\end{array}$ & Não apresentada & Não apresentada & $\begin{array}{l}\text { Em 86,1\% dos casos, a dor deveria ter sido avaliada } \\
\text { pelo enfermeiro, mas isso não aconteceu. A } \\
\text { existência de outros procedimentos hospitalares } \\
\text { junto ao cliente durante a triagem (exemplo: coleta } \\
\text { de sangue),, automedicação analgésica antes do } \\
\text { atendimento na UUE (o enfermeiro tinha o pré- } \\
\text { conceito de que esse fato prejudicava a avaliação } \\
\text { fidedigna da dor) e o uso do protocolo de } \\
\text { Manchester, que limitava a anamnese e exame } \\
\text { físico, não focando os aspectos importantes na } \\
\text { avaliação de dor, prejudicaram a avaliação. }\end{array}$ \\
\hline $\begin{array}{l}\text { Ribeiro et al. } \\
\text { Brasil } \\
2011 \\
\text { IV }\end{array}$ & $\begin{array}{l}\text { Os enfermeiros avaliavam a dor por } \\
\text { meio da observação de } \\
\text { comportamentos de dor do cliente } \\
\text { (choro, expressão facial, posição } \\
\text { antálgica, agitação, alteração no } \\
\text { padrão do sono, impaciência, } \\
\text { hipoatividade, inquietação) e de } \\
\text { dados objetivos (sinais vitais, } \\
\text { sudorese e palidez). }\end{array}$ & $\begin{array}{l}\text { Administrar analgésicos conforme } \\
\text { prescrição médica e realizar terapêuticas } \\
\text { analgésicas não farmacológicas, como } \\
\text { diálogo, orientação, posicionamento } \\
\text { adequado no leito, conforto térmico, } \\
\text { retirada de fraldas molhadas e sujas, } \\
\text { banho, uso de compressas locais, } \\
\text { exercícios de respiração, massagens, } \\
\text { atitudes humanizadas, técnicas de } \\
\text { relaxamento e distração. }\end{array}$ & Não apresentada & Não apresentadas \\
\hline $\begin{array}{l}\text { Nascimento e Kreling }^{(4)} \\
\text { Brasil } \\
2011 \\
\text { IV }\end{array}$ & $\begin{array}{c}\text { Técnicos e auxiliares de enfermagem } \\
\text { avaliavam a presença de dor por } \\
\text { meio da escala de descritores verbais } \\
\text { ou Escala Numérica, durante o } \\
\text { momento da avaliação dos sinais } \\
\text { vitais }\end{array}$ & Não apresentada & Não apresentada & $\begin{array}{l}\text { Técnicos e auxiliares de enfermagem relataram } \\
\text { dificuldades em avaliar a dor, devido à falta de } \\
\text { compreensão das escalas de intensidade de dor por } \\
\text { parte do paciente e por falta de tempo para avaliá- } \\
\text { la. Foram apontadas as limitações do enfermeiro } \\
\text { enquanto supervisor e gestor da dor do cliente, com } \\
\text { base nos conhecimentos e atitudes pró-ativas. }\end{array}$ \\
\hline $\begin{array}{l}\text { Shaban et al. } \\
\text { Austrália } \\
2012 \\
\text { IV }\end{array}$ & Não apresentada & $\begin{array}{l}\text { Utilizar o fluxograma de indicação } \\
\text { analgésica conforme protocolo da } \\
\text { instituição, no serviço de triagem. }\end{array}$ & Não apresentada & $\begin{array}{l}\text { Enfermeiros relataram dificuldades na implantação } \\
\text { do protocolo de indicação analgésica na triagem, } \\
\text { devido à reduzida valorização às informações } \\
\text { subjetivas do cliente, falta de empatia e pressões } \\
\text { por parte da empresa. }\end{array}$ \\
\hline
\end{tabular}




\begin{tabular}{|c|c|c|c|c|}
\hline \multirow{2}{*}{$\begin{array}{c}\text { Autores/País/Ano/nível } \\
\text { de evidência }\end{array}$} & \multicolumn{3}{|c|}{ Manejo da dor } & \multirow{2}{*}{ Barreiras no manejo da dor } \\
\hline & Avaliação & Intervenção & Monitorização & \\
\hline $\begin{array}{l}\text { Fry, Hearn, } \\
\text { McLaughlin }^{(14)} \\
\text { Austrália } \\
2012 \\
\text { IV }\end{array}$ & $\begin{array}{l}\text { Os enfermeiros do serviço de } \\
\text { triagem avaliavam a dor por meio da } \\
\text { EN, escala de descritores verbais e } \\
\text { questionamentos quanto ao local de } \\
\text { dor. }\end{array}$ & $\begin{array}{c}\text { Aplicar o protocolo local para prescrição } \\
\text { analgésica pelo enfermeiro ainda na } \\
\text { triagem. }\end{array}$ & Não apresentada & $\begin{array}{l}\text { O fato de o cliente ter praticado a automedicação } \\
\text { analgésica antes do atendimento na UUE foi barreira } \\
\text { para administração de outros analgésicos, pelo } \\
\text { temor de ocorrência de eventos adversos. Apenas } \\
47 \% \text { dos enfermeiros utilizavam o fluxograma de } \\
\text { indicação analgésica, proposto pelo protocolo da } \\
\text { instituição. }\end{array}$ \\
\hline $\begin{array}{l}\text { Bergman }^{(24)} \\
\text { Estados Unidos da } \\
\text { América } \\
2012 \\
\text { IV }\end{array}$ & $\begin{array}{l}\text { Enfermeiros do serviço de urgência e } \\
\text { emergência de um hospital de } \\
\text { grande porte referiram uso } \\
\text { frequente da Escala Numérica de } \\
\text { dor. }\end{array}$ & Não apresentada & Não apresentada & $\begin{array}{l}\text { Sobrecarga de trabalho inerente às demandas do } \\
\text { setor (grande número de pacientes aguardando } \\
\text { atendimento, insuficiência de leitos para } \\
\text { observação, gerando a priorização de quadros } \\
\text { clínicos graves em detrimento de clientes que } \\
\text { apenas queixam dor), conflitos no ambiente de } \\
\text { trabalho (desempenho de atividades voltadas ao } \\
\text { cuidar e gerenciais concomitantemente, gerando } \\
\text { cobranças da gestão quanto ao desempenho do } \\
\text { enfermeiro; discórdia com profissionais médicos que } \\
\text { não avaliam e prescrevem rapidamente a clientes já } \\
\text { avaliados com dor pelo enfermeiro - falta de } \\
\text { autonomia quanto à gestão do cliente com dor) e } \\
\text { frustração quanto ao papel do enfermeiro nas UUEs } \\
\text { que, devido aos fatores acima, deixa a desejar no } \\
\text { desempenho de suas funções. }\end{array}$ \\
\hline $\begin{array}{l}\text { Silva et al. }{ }^{(13)} \\
\text { Brasil } \\
2013 \\
\text { IV }\end{array}$ & $\begin{array}{l}\text { Os enfermeiros utilizavam o } \\
\text { protocolo de Manchester, que avalia } \\
\text { a dor quanto à localização e } \\
\text { intensidade. }\end{array}$ & Não apresentada & Não apresentada & $\begin{array}{l}\text { A restrição do enfermeiro quanto ao uso do } \\
\text { protocolo de Manchester limitou a avaliação } \\
\text { adequada do fenômeno doloroso nas UUEs. }\end{array}$ \\
\hline $\begin{array}{l}\text { Gonçalves et al. }{ }^{(21)} \\
\text { Brasil } \\
2013 \\
\text { IV }\end{array}$ & $\begin{array}{l}\text { Enfermeiros e técnicos em, } \\
\text { enfermagem avaliavam a dor em } \\
\text { uma UUE infantil, por meio de } \\
\text { alterações no comportamento da } \\
\text { criança (expressão facial, choro, } \\
\text { postura, feição) e relato verbal, } \\
\text { quando possível. }\end{array}$ & $\begin{array}{l}\text { Administrar analgésicos conforme } \\
\text { prescrição médica e utilizar terapêuticas } \\
\text { não farmacológicas, como sucção não } \\
\text { nutritiva, pegar no colo e compressas de } \\
\text { calor e frio. }\end{array}$ & Não apresentada & Não apresentadas \\
\hline
\end{tabular}




\begin{tabular}{|c|c|c|c|c|}
\hline \multirow{2}{*}{$\begin{array}{c}\text { Autores/País/Ano/nível } \\
\text { de evidência }\end{array}$} & \multicolumn{3}{|c|}{ Manejo da dor } & \multirow{2}{*}{ Barreiras no manejo da dor } \\
\hline & Avaliação & Intervenção & Monitorização & \\
\hline $\begin{array}{c}\text { Ucuzal e Dogan }^{(22)} \\
\text { Turquia } \\
2015 \\
\text { IV }\end{array}$ & $\begin{array}{l}\text { A maior parte }(80,7 \%) \text { dos } \\
\text { enfermeiros investigados ( } n=57) \text { não } \\
\text { utilizava escalas de dor para avaliá-la } \\
\text { durante a triagem. Quase metade } \\
(48,4 \%) \text { baseava sua avaliação de dor } \\
\text { em comportamentos do cliente. }\end{array}$ & $\begin{array}{c}\text { Administrar analgésicos prescritos como } \\
\text { "se necessário", quando solicitado pelo } \\
\text { cliente. }\end{array}$ & Não apresentada & $\begin{array}{l}\text { Medo da administração de superdosagens de } \\
\text { analgésicos e déficit no conhecimento sobre } \\
\text { avaliação da dor. }\end{array}$ \\
\hline $\begin{array}{l}\text { Pretorius, Searle, } \\
\text { Marshall }^{(26)} \\
\text { Nova Zelândia } \\
2015 \\
\text { IV }\end{array}$ & Não apresentada & Não apresentada & Não apresentada & $\begin{array}{l}\text { Sobrecarga de responsabilidades do enfermeiro, } \\
\text { morosidade entre a avaliação de dor pelo } \\
\text { enfermeiro e a intervenção médica, relutância do } \\
\text { paciente em reportar sua dor; pacientes que, } \\
\text { anteriormente ao atendimento, fizeram uso de } \\
\text { álcool ou outras drogas, conhecimento inadequado } \\
\text { do enfermeiro sobre os princípios para o manejo da } \\
\text { dor, monitorização inadequada do alívio da dor e } \\
\text { relutância do cliente em usar opioides. }\end{array}$ \\
\hline $\begin{array}{l}\text { Dale e Bjørnsen }^{(25)} \\
\text { Noruega } \\
2015 \\
\text { IV }\end{array}$ & $\begin{array}{l}\text { Enfermeiros do serviço de triagem da } \\
\text { UUE usavam a EN. No estudo, } 77 \% \\
\text { dos clientes atendidos tiveram a dor } \\
\text { avaliada. Os que não foram avaliados } \\
\text { quanto à dor foi devido ao fato de } \\
\text { apresentarem alterações clínicas } \\
\text { mais prioritárias. }\end{array}$ & $\begin{array}{l}\text { Administrar analgésicos conforme } \\
\text { prescrição médica. }\end{array}$ & Não apresentada & Não apresentadas \\
\hline
\end{tabular}




\section{DISCUSSÃO}

Para análise, os achados foram agrupados em três grandes eixos que compilam as evidências de forma a facilitar a sua discussão, portanto, integram: a avaliação da dor pela equipe de enfermagem, as intervenções utilizadas para alívio da dor e o monitoramento da dor, para cada um dos tópicos foram abordadas as barreiras identificadas na revisão.

\section{Avaliação da dor pela equipe de enfermagem}

Dificuldades na avaliação da dor foram evidenciadas, sugerindo que os profissionais de enfermagem não avaliam a dor conforme recomendado ${ }^{(6,15)}$, ou seja, não fazem avaliação de local, intensidade, início, duração, frequência, qualidade sensitiva, fatores que agravam e atenuam a dor, impacto gerado, bem como de seu padrão evolutivo ${ }^{(28)}$.

Sobre os instrumentos de avaliação de dor, os profissionais de enfermagem optaram pelas escalas numéricas, de descritores verbais e as visuais analógicas ${ }^{(4-5,14,20,23)}$. No entanto, os profissionais afirmaram utilizá-las pouco, lançando mão de parâmetros isolados ${ }^{(7,10,13,15,21,27)}$, o que prejudica a fidedignidade do processo $^{(21)}$.

Estudos $^{(4-5,20)}$ mostraram que os profissionais têm dificuldade em utilizar as escalas de avaliação, seja pelo desconhecimento, seja pela dificuldade de interpretação dos instrumentos pelos clientes. Estudo ${ }^{(4)}$ que investigou o comportamento de auxiliares e técnicos de enfermagem frente ao manejo da dor evidenciou dificuldade dos profissionais na interpretação dos instrumentos de medição de dor.

Nesse sentido, a má interpretação ou subestimação da dor resultarão em intervenção inadequada ${ }^{(29)}$. Utilizar instrumentos multidimensionais válidos para a população e cultura em questão e capazes de abranger os principais aspectos da dor pode contribuir ${ }^{(30)}$. No entanto, mediante a exigência de avaliações sucintas para intervenções precoces, recomenda-se que, minimamente, as escalas numéricas ou de descritores verbais sejam utilizadas nas UUEs ${ }^{(1,7,30)}$. A avaliação adequada da dor contribui para o seu tratamento, pois é por meio dela que se define a necessidade de novas intervenções, avalia-se a eficácia da intervenção prescrita ou se decide pela suspensão daquela implementada.

$O$ afastamento do enfermeiro do processo avaliativo e de gestão da dor foi evidenciado ${ }^{(4)}$. No entanto, tais profissionais têm o dever legal de exercer liderança diante dos diferentes fenômenos vivenciados por seus clientes ${ }^{(31)}$. A ausência de boa gestão da dor pelo líder da equipe pode ser a causa das dificuldades referidas por parte dos técnicos e auxiliares de enfermagem no uso de escalas para avaliação da dor.

Sobre as dificuldades para avaliação da dor, os profissionais colocaram barreiras, como a sobrecarga de trabalho e as pressões do serviço advindas da exigência de utilizar protocolos para o manejo da dor ${ }^{(4,20)}$. A classe de enfermagem constantemente se submete à cargas horárias excessivas, trabalhando sem planejamento em suas atividades rotineiras, o que gera desgaste e fadiga pelas prolongadas jornadas de trabalho ${ }^{(32)}$. Todavia, o manejo adequado da dor não pode ficar comprometido por questões trabalhistas, é necessário desenvolver conhecimentos, habilidades e atitudes a fim de melhorar a prática de manejo da dor, 
mesmo em meio aos desafios da profissão ${ }^{(33)}$.

Intervenção para alívio da dor pela equipe de enfermagem

Na Austrália, África e Jerusalém, os clientes receberam analgesia ainda na triagem, conforme indicação do enfermeiro, que se vale de protocolos/algoritmos governamentais e institucionais que respaldam a prescrição de analgésicos por esses profissionais até haver a definição da conduta médica ${ }^{(5-6,14,20,23)}$.

No Brasil, o processo de analgesia medicamentosa nas UUEs ainda se restringe à decisão médica no momento do atendimento ${ }^{(1)}$. No entanto, a lei do exercício profissional de enfermagem assegura ao enfermeiro do Brasil a prescrição de medicamentos aprovados nos programas de saúde pública e em rotinas das instituições ${ }^{(31)}$. Assim, para que haja melhoria na analgesia de clientes atendidos na triagem e minimização do tempo de espera para recebê-la, são necessárias a reformulação das políticas públicas de saúde sobre o tema, a sensibilização e mudança de cultura do governo e da sociedade e a capacitação dos profissionais de enfermagem para essa tarefa, além da readequação da formação de enfermeiros, incluindo a revisão de currículos das instituições de ensino.

Minimizar rapidamente o sofrimento do cliente é uma questão ética(2) e de humanização da assistência $^{(12)}$. O enfermeiro pode valer-se de outras estratégias, como apoderar-se da Política Nacional de Humanização ${ }^{(34)}$, com acolhimento e escuta qualificada dos indivíduos que buscam atendimento em UUEs e esperam o alívio de sua dor. Tal conduta poderia auxiliar a superar a barreira encontrada no presente estudo quanto à falta de empatia do profissional com a dor do cliente.

Terapêuticas não farmacológicas podem ser úteis, destacando-se as ações em UUE pediátrica. Os cuidados de enfermagem mais utilizados nessas situações foram: suç̧ão não nutritiva, pegar no colo, calor e frio locais ${ }^{(21)}$. Em UUE de adultos, também foram elencadas ações, como: estabelecer diálogo com o cliente, realizar orientações, posicionar o cliente adequadamente no leito, oferecer conforto térmico, retirar fraldas molhadas ou sujas, realizar ou auxiliar no banho, usar compressas locais, estimular exercícios de respiração, realizar massagens, proporcionar um ambiente confortável, atender com humanização, promover alívio de pressão em pele, dar apoio emocional com esclarecimentos, ensinar técnicas de relaxamento e distração, oferecer conforto verbal, entre outras ações ${ }^{(20)}$. No entanto, foram pouco utilizadas nas UUEs, talvez pela urgência em aliviar a dor nessas unidades.

A conduta dos enfermeiros para intervir em clientes com queixa de dor foi significativamente melhorada após a participação em programas de educação continuada ou capacitações ${ }^{(23,27)}$. O manejo adequado da dor nas UEEs pode ser alcançado mediante desenvolvimento dos profissionais a partir de capacitações, treinamentos e cursos emergenciais ou pontuais, contínuos e estruturados ${ }^{(35)}$.

\section{Monitoramento da dor}

No monitoramento da dor, evidenciaram-se os aspectos objetivos (escalas de avaliação de dor, sinais vitais, presença de agitação, fácies de dor e conforto) e subjetivos (relato de dor) ${ }^{(10)}$. Outro estudo ${ }^{(23)}$ deixou 
evidente o uso de dados objetivos, como o uso de escalas de avaliação de dor, reforçando a importância do uso delas.

Salienta-se que a falta de monitorização da dor após a administração de terapêuticas analgésicas coloca em risco o sucesso do tratamento oferecido para alívio da dor, expondo o paciente a sofrimento desnecessário.

\section{CONCLUSÃO}

Os clientes com dor de intensidade elevada ainda esperam o alívio nas UUEs. Tal fato poderia ser minimizado pelo uso de protocolos e fluxogramas baseados em programas governamentais de saúde pública que permitem ao enfermeiro realizar a analgesia precocemente.

Barreiras que impedem o manejo adequado da dor são reais. Dentre elas, destacam-se a dificuldade da equipe e dos clientes em interpretar as escalas de avaliação, o distanciamento do enfermeiro da prática de gestão da dor e a sobrecarga de trabalho dos profissionais de enfermagem.

Há necessidade de sensibilizar e capacitar os profissionais de enfermagem quanto ao manejo da dor, empoderando o enfermeiro nesse processo, bem como a revisão da legislação brasileira em urgência e emergência para que a dor seja incluída como prioridade nas UUEs são desejadas.

Os achados evidenciam necessidade de reorganização da assistência de enfermagem oferecida às pessoas com dor que procuram atendimento nas UUEs, apontando o manejo da dor como ponto fundamental para a humanização da assistência e qualidade do atendimento.

\section{REFERÊNCIAS}

1. Calil AM. Dor e Analgesia em Vítimas de acidentes de transporte atendidas em um pronto socorro [tese]. São Paulo. Escola de Enfermagem. Universidade de São Paulo. 2003. 182 p.

2. Souza LAF, Pessoa AP da C, Barbosa MA, Pereira LV. O modelo bioético principialista aplicado no manejo da dor. Rev Gaúcha Enferm. 2013;34(1):187-95.

3. Sallum AMC, Garcia DM, Sanches M. Acute and chronic pain: a narrative review of the literature. Acta Paul Enferm. 2012;25(spe1):150-4.

4. Nascimento LA, Kreling MCGD. Avaliação da dor como quinto sinal vital: opinião de profissionais de enfermagem. Acta Paul Enferm. 2011;24(1):50-4.

5. Rampanjato RM, Florence M, Patrick NC, Finucane BT. Factors influencing pain management by nurses in emergency departments in Central Africa. Emerg Med J. 2007;24(7):475-6.

6. van der Wulp I, Sturms LM, Jong A, Schot-Balfoort M, Schrijvers AJP, van Stel HF. Pain assessments at triage with the Manchester triage system: a prospective observational study. Emerg Med J. 2011;28(7):585-9.

7. Calil AM, Pimenta CA de M. Importância da avaliação e padronização analgésica em serviços de emergência. Acta Paul Enferm. 2010;23(1):53-9.

8. Castilho-Bueno M, Moreno-Pina J, Martinez-Puente M, Artiles-Suaréz M, Company-Sacho M, García-Andrés M, et al. Effectiveness of nursing intervention for experiencing chronic pain : a systematic review adult patients Executive summary. JBI Libr Syst Rev. 2010;8(28):1112-68.

9. Dunwoody CJ, Krenzischek DA, Pasero C, Rathmell JP, Polomano RC. Assessment, physiological monitoring, and consequences of inadequately treated acute pain. J Perianesth Nurs. 2008;23(1 Suppl):S15-27.

10. Calil AM, Pimenta CA de M. Conceitos de enfermeiros e médicos de um serviço de emergência sobre dor e analgesia no trauma. Rev da Esc Enferm da USP; 2005;39(3):325-32. 
11. Bottega FH, Fontana RT. A dor como quinto sinal vital: utilização da escala de avaliação por enfermeiros de um hospital geral. Texto Context - Enferm. 2010;19(2):283-90.

12. Calil AM. Avaliação da Dor e Analgesia no Atendimento Pré-hospitalar em Pacientes de Trauma. Rev. Gaúcha Enferm. 2008; 29(2):308-13.

13. Silva AP da, Diniz AS, Araújo FA, Souza CC de. Presencia de la queja de dolor en pacientes clasificados de acuerdo con el Protocolo de Manchester. Rev Enferm Cent-Oeste Min. 2013;3(1):507-17.

14. Fry M, Hearn J, McLaughlin T. Pre-hospital pain management patterns and triage nurse documentation. Int Emerg Nurs. 2012;20(2):83-7.

15. Shaban RZ, Holzhauser K, Gillespie K, Huckson S, Bennetts S. Characteristics of effective interventions supporting quality pain management in Australian emergency departments: an exploratory study. Australas Emerg Nurs J. 2012;15(1):23-30.

16. Pereira AL, Bachion MM. Atualidades em revisão sistemática de literatura, critérios de força e grau de recomendação de evidência. Rev Gaúcha Enferm. 2006. 27(4):491-8.

17. Baldini Soares C, Akiko Komura Hoga L, Peduzzi M, Sangaleti C, Yonekura T, Rachel Audebert Delage Silva D. Integrative review: concepts and methods used in nursing descriptors Integrative review: concepts and methods used in nursing Critical review. Rev Esc Enferm USP. 2014;48(2):329-39.

18. Biruel P, Pinto R. Bibliotecário: Um profissional a serviço da pesquisa. In: Anais do XXIV Congresso Brasileiro de Biblioteconomia, Documentação e Ciência da Informação. Maceió, Alagoas, Brasil; 2011.

19. Stetler CB, Brunell M, Giuliano KK, Morsi D, Prince L, Newell-Stokes V. Evidence-based practice and the role of nursing leadership. J Nurs Adm. 1998 ;28(7-8):45-53.

20. Ribeiro NCA, Barreto SCC, Hora EC, Sousa RMC de. O enfermeiro no cuidado à vítima de trauma com dor: o quinto sinal vital. Rev da Esc Enferm da USP. 2011;45(1):146-52.

21. Gonçalves B, Holz AW, Lange C, Maagh SB, Pires CG, Brazil CM. O cuidado da criança com dor internada em uma unidade de emergência e urgência pediátrica. Rev Dor. 2013;14(3):179-83.

22. Ucuzal M, Doğan R. Emergency nurses' knowledge, attitude and clinical decision making skills about pain. Int Emerg Nurs. 2015;23(2):75-80.

23. Stalnikowicz R, Mahamid R, Kaspi S, Brezis M. Undertreatment of acute pain in the emergency department: a challenge. Int J Qual Health Care. 2005;17(2):173-6.

24. Bergman CL. Emergency Nurses' Perceived Barriers to Demonstrating Caring When Managing Adult Patients' Pain. J Emerg Nurs. Emergency Nurses Association; 2012;38(3):218-25.

25. Dale J, Bjørnsen LP. Assessment of pain in a Norwegian Emergency Department. Scand J Trauma Resusc Emerg Med. Scandinavian Journal of Trauma, Resuscitation and Emergency Medicine; 2015;23(1):86.

26. Pretorius A, Searle J, Marshall B. Barriers and Enablers to Emergency Department Nurses' Management of Patients' Pain. Pain Manag Nurs. American Society for Pain Management Nursing; 2015;16(3):1-8.

27. Le May S, Johnston CC, Choinière M, Fortin C, Kudirka D, Murray L, et al. Pain Management Practices in a Pediatric Emergency Room (PAMPER) Study: interventions with nurses. Pediatr Emerg Care. 2009;25(8):498-503.

28. Pimenta CA de M, Koizumi MS, Teixeira MJ. Dor, depressão e conceitos culturais. Arq Neuropsiquiatr. 1997;55(3A):370-80.

29. Sousa FAEF, Pereira LV, Cardoso R, Hortense P. Multidimensional pain evaluation scale. Rev Lat Am Enfermagem. 2010;18(1):03-10.

30. Sousa FAEF, Pereira LV, Hortense P. Avaliação e mensuração da percepção de dor. In: Alves Neto O, Costa CM de C, Siqueira JTT, Teixeira MJ, editors. Dor: Princípios e Práticas. Fisrt. Porto Alegre: Artmed; 2009. p. 370-81.

31. Brasil. Lei n. 7498, de 25 de junho de 1986. Dispõe sobre a regulamentação do Exercício da Enfermagem e dá outras providências. Brasília-DF.: Diário Oficial da República Federativa do Brasil; 1986.

32. Silva DB, Balbino CM. Fatores que contribuem para a sobrecarga de trabalho e seus efeitos no desempenho profissional do enfermeiro: revisão literária [Internet]. Revista Pró-UniverSUS. 2015 [cited 2016 Jan 3]. p. 11. Available from: http://editorauss.uss.br/index.php/RPU/article/view/384.

33. Peres AM, Ciampone MHT. Gerência e competências gerais do enfermeiro. Texto Context - Enferm. 2006;15(3):492-9.

34. Brasil, Ministério da Saúde. Política Nacional de Humanização. Brasília-DF 1a edição. [internet]. 2013 [acesso em: 20 mar 2015]. Disponível em:

http://bvsms.saude.gov.br/bvs/publicacoes/politica_nacional_humanizacao_pnh_folheto.pdf.

Rev. Eletr. Enf. [Internet]. 2016 [acesso em:_____];18:e1171. Disponível em: http://dx.doi.org/10.5216/ree.v18.37309. 
35. Silva GM da, Seiffert OMLB. Educação continuada em enfermagem: uma proposta metodológica. Rev Bras Enferm. 2009;62(3):362-6. 